\title{
Relative telomere length and oxidative DNA damage in hypertrophic ligamentum flavum of lumbar spinal stenosis
}

\author{
Sinsuda Dechsupa ${ }^{1}$, Wicharn Yingsakmongkol ${ }^{2}$, Worawat Limthongkul ${ }^{2}$, Weerasak Singhatanadgige ${ }^{2}$, \\ Sittisak Honsawek ${ }^{\text {Corresp. } 1}$ \\ ${ }^{1}$ Osteoarthritis and Musculoskeleton Research Unit, Department of Biochemistry, Faculty of Medicine, King Chulalongkorn Memorial Hospital, Thai Red \\ Cross Society, Chulalongkorn University, Bangkok, Thailand \\ 2 Department of Orthopaedics, Faculty of Medicine, King Chulalongkorn Memorial Hospital, Thai Red Cross Society, Chulalongkorn University, Bangkok, \\ Thailand \\ Corresponding Author: Sittisak Honsawek \\ Email address: sittisak.h@chula.ac.th
}

Background. Lumbar spinal stenosis (LSS) is a common cause of low back pain with degenerative spinal change in older adults. Telomeres are repetitive nucleoprotein DNA sequences of TTAGGG at the ends of chromosomes. Oxidative stress originates from an imbalance in pro-oxidant and antioxidant homeostasis that results in the production of reactive oxygen species (ROS). The purpose of this study was to investigate relative telomere length (RTL) and oxidative damage in ligamentum flavum (LF) tissue from LSS patients. Methods. Forty-eight patients with LSS participated in this study. Genomic DNA from non-hypertrophic and hypertrophic LF tissue was analyzed by real time-polymerase chain reaction for relative telomere length (RTL). 8-hydroxy 2'-deoxygaunosine (8-OHdG) levels were determined by using enzyme-linked immunosorbent assay. We cultivated LF fibroblast cells from patients in different ages $(61,66$, and 77 years). After each cultivation cycle, we examined RTL and senescence-associated $\beta$-galactosidase (SA- $\beta$-gal) expression. Results. The hypertrophic LF had significantly lower RTL than nonhypertrophic LF $(P=0.04)$. The levels of $8-O H d G$ were significantly higher in hypertrophic LF compared to non-hypertrophic LF $(P=0.02)$. With advancing cell culture passage, the number of cells in each passage was significantly lower in hypertrophic LF fibroblast cells than non-hypertrophic LF fibroblast cells. When evaluated with SA- $\beta$-gal staining, all senescent LF fibroblast cells were observed at earlier passages in hypertrophic LF compared with non-hypertrophic LF fibroblast cells. Discussion. Our results showed that patients with LSS displayed an accelerated RTL shortening and high oxidative stress in hypertrophic LF. These findings implied that telomere shortening and oxidative stress may play roles in the pathogenesis of hypertrophic LF in lumbar spinal stenosis. 
1 Manuscript Title:

2

3 Relative telomere length and oxidative DNA damage in hypertrophic ligamentum flavum

4 of lumbar spinal stenosis

5 Authors:

6 Sinsuda Dechsupa ${ }^{1}$, Wicharn Yingsakmongkol ${ }^{2}$, Worawat Limthongkul ${ }^{2}$, Weerasak

7 Singhatanadgige ${ }^{2}$, Sittisak Honsawek ${ }^{1, *}$

8 Affiliations:

$9{ }^{1}$ Osteoarthritis and Musculoskeleton Research Unit, Department of Biochemistry, Faculty of

10 Medicine, Chulalongkorn University, King Chulalongkorn Memorial Hospital, Thai Red Cross

11 Society, Bangkok, Thailand

$12{ }^{2}$ Department of Orthopaedics, Faculty of Medicine, Chulalongkorn University, King

13 Chulalongkorn Memorial Hospital, Thai Red Cross Society, Bangkok, Thailand

14 Corresponding Author:

15 Sittisak Honsawek.

16 E-mail: $\underline{\text { sittisak.h@chula.ac.th }}$ 


\section{Abstract}

18 Background. Lumbar spinal stenosis (LSS) is a common cause of low back pain with

19 degenerative spinal change in older adults. Telomeres are repetitive nucleoprotein DNA sequences of TTAGGG at the ends of chromosomes. Oxidative stress originates from an imbalance in pro-oxidant and antioxidant homeostasis that results in the production of reactive oxygen species (ROS). The purpose of this study was to investigate relative telomere length (RTL) and oxidative damage in ligamentum flavum (LF) tissue from LSS patients.

Methods. Forty-eight patients with LSS participated in this study. Genomic DNA from nonhypertrophic and hypertrophic LF tissue was analyzed by real time-polymerase chain reaction for relative telomere length (RTL). 8-hydroxy 2'-deoxygaunosine (8-OHdG) levels were determined by using enzyme-linked immunosorbent assay. We cultivated LF fibroblast cells from patients in different ages (61, 66, and 77 years). After each cultivation cycle, we examined RTL and senescence-associated $\beta$-galactosidase (SA- $\beta$-gal) expression.

Results. The hypertrophic LF had significantly lower RTL than non-hypertrophic LF $(P=0.04)$. The levels of 8-OHdG were significantly higher in hypertrophic LF compared to nonhypertrophic LF $(P=0.02)$. With advancing cell culture passage, the number of cells in each passage was significantly lower in hypertrophic LF fibroblast cells than non-hypertrophic LF fibroblast cells. When evaluated with SA- $\beta$-gal staining, all senescent LF fibroblast cells were 
35 observed at earlier passages in hypertrophic LF compared with non-hypertrophic LF fibroblast

36 cells.

37 Discussion. Our results showed that patients with LSS displayed an accelerated RTL shortening

38 and high oxidative stress in hypertrophic LF. These findings implied that telomere shortening

39 and oxidative stress may play roles in the pathogenesis of hypertrophic LF in lumbar spinal

40 stenosis.

41

42

43

44

45

46

47

48

49

50 


\section{Introduction}

52 Lumbar spinal stenosis (LSS) is one of the most common spinal pathologies in the elderly and

53 results in buttock or lower extremity pain, radiculopathy, neurogenic claudication, and cauda

54 equina syndrome. LSS has been one of the leading indications for lumbar spinal surgery in

55 patients older than 60 years of age. The finding of LSS is characterized by a decrease of elastin-

56 to-collagen ratio, age-related fibrosis, and hypertrophic ligamentum flavum (Katz et al., 2008).

57 Ligamentum flavum (LF) is a yellow ligament containing high collagen and elastin fibers.

58 Elastin provides the elasticity, while collagen fiber provides the tensile strength and stability of

59 the spine (Losiniecki et al., 2013; Li et al., 2015; Yabe et al., 2015). With age and degeneration,

60 an increase in collagen and a decreased elastin-to-collagen ratio were evident in hypertrophied

61 LF (Mobbs et al., 2007); however, the underlying mechanisms remain unknown. Environmental,

62 biochemical, and genetic risk factors may be involved in the pathogenesis of LSS. A number of

63 cytokines have been postulated to play major parts in the fibrosis of LF in LSS. In recent years,

64 the increased expressions of various matrix metalloproteases, connective tissue growth factor,

65 basic fibroblast growth factor, and inflammatory cytokines have been highlighted in the

66 hypertrophy of LF (Park et al., 2009; Zhong et al., 2011; Honsawek et al., 2013; Park et al.,

67 2013). Telomere length and oxidative stress including DNA damage have never been

68 investigated in lumbar spinal stenosis. 

round of cell proliferation both in vitro and in vivo due to the inabilities of conventional polymerases to fully replicate the parenting DNA by lagging strand synthesis (the end replication problem) (O'Sullivan et al., 2010 ). Eventually, this can lead to telomere shortening and cell cycle arrest at G1 phase, which consequence to senescent cells and apoptosis (Zhu et al., 2011). One senescence marker is senescence-associated $\beta$-galactosidase ( $\mathrm{SA}-\beta$-gal) staining, which increases with cellular aging (Kim et al., 2008; Kim et al., 2009; Takahashi et al., 2014). Several studies have shown telomere shortening in various conditions including degenerated intervertebral discs, keloid, and biliary atresia (Le Maitre et al., 2007; De Felice et al., 2009;

Udomsinprasert et al., 2015). nitrosative species (RNS) ( $L$ u et al., 2007). Moreover, exogenous factors can produce ROS and/or RNS, including UV radiation, smoking, and carcinogenic substances (Droge, 2002). High

86 levels of ROS have been demonstrated as oxidative damage to lipids of cell membranes,

87 proteins, and DNA damage to human cells (Valavanidis et al., 2009). Oxidative DNA damage 
88 has been produced by hydroxyl radical $\left(\mathrm{OH}^{\circ}\right)$ from various mechanisms and lead to the

89 interaction of $\mathrm{OH}^{\bullet}$ with genomic base, which is forming 8-hydroxy 2'-deoxyguanosine (8-

90 OHdG) (Valko et al., 2004; Kroese et al., 2014). 8-OHdG can be a biomarker for oxidative

91 stress in carcinogenesis, aging, and cell senescence and can be found in human samples, such as

92 blood leukocytes, tissues, and urine (Sajous et al., 2000). LSS may be induced by oxidative

93 stress, leading to DNA damage and nucleobase instability. Until now, the possible roles of

94 telomere length and oxidative DNA damage, including the SA- $\beta$-gal for cell senescence, have

95 never been investigated in LSS patients.

97 conditions and cellular aging. We hypothesized that telomere shortening and oxidative DNA damage could be associated with the hypertrophic LF in LSS patients. Accordingly, the objectives of this study were to investigate relative telomere length (RTL) and oxidative DNA patients of different age for in vitro cell culture to determine RTL and evaluate senescence markers as $\mathrm{SA}-\beta-$ gal expression.

\section{Study population}


106 LF specimens were obtained from 48 lumbar spinal stenosis patients (19 male and 29 female)

107 who underwent decompression laminectomy for neurogenic claudication. The mean age of

108 patients was 63.3 \pm 9.7 years (range, 45.0-87.0 years). The hypertrophic LF specimens were

109 obtained from hypertrophic LF levels in each patient which mostly occurred at L3/L4 and L4/L5.

110 The non-hypertrophic LF was collected from the same patients who served as a control group.

111 All LF specimens were pooled in $1.5 \mathrm{~mL}$ microcentrifuge tubes containing $200 \mu \mathrm{L}$ of RNAlater

112 (Qiagen, Hilden, Germany) and kept at $-80^{\circ} \mathrm{C}$ until measurement. All human LF specimens were

113 obtained with the written informed consent of the patients who participated in this study. All of

114 the consent procedure and experimental protocols were approved by the Institutional Review

115 Board on Human Research of the Faculty of Medicine, Chulalongkorn University (IRB 259/59).

\section{Isolation and culture of human LF cells}

117 Hypertrophic and non-hypertrophic LF specimens were classified into three age groups as

118 follows 61, 66, and 77 years. LF cells were isolated as described in a previous study ( $S W$ et al.,

119 2014). Whole LF tissue was minced into pieces of approximately $0.2 \mathrm{~cm}$ into T25 flasks. Cells

120 were maintained in complete medium (Alpha-Modified Eagle's medium with 10\% fetal bovine

121 serum, 1\% penicillin-streptomycin [GIBCO - BRL, Grand Island, NY, USA]) at $37^{\circ} \mathrm{C}$ in

122 humidified atmosphere containing $5 \% \mathrm{CO}_{2}$ until they grew to confluence. Cells were fed by 
123 complete replacement of medium every 3 days. When the primary cultures reached confluence,

124 adherent cells were detached using $0.25 \%$ trypsin EDTA solution (GIBCO - BRL) and seeded at

125 a density of $1.0 \times 10^{5}$ cells in T25 flasks containing complete medium. When reaching

126 confluence, cells were trypsinized and passaged sequentially in the same manner until 10

127 passages. Throughout the experiment, human LF cells were cultivated under the standard culture

128 condition.

129 Determination of relative telomere length

130 Genomic DNA was extracted directly from LF tissues and cells according to the instruction of

131 DNA extraction kit (Vivantis, Buckinghamshire, Malaysia). The concentration of DNA samples

132 was measured using the Nanodrop 2000 spectrophotometer (Thermo Scientific, Wilmington, DE,

133 USA). The relative telomere length was determined by quantitative real-time PCR method, as

134 previously described by Cawthon (Cawthon, 2002). Telomere length was measured according to

135 the ratio of the telomere repeat copy number (T) to the single-copy gene copy number (S) in each

136 given sample. The single-copy gene refers to the 36B4 gene, which encodes the acid ribosomal

137 phosphoprotein $(\mathrm{PO})$. The ratio $(\mathrm{T} / \mathrm{S})$ is proportional to the average telomere length. To begin

138 with, the RTL was analyzed by using SYBRGreen Master Mix none-ROX (RBC Bioscience,

139 Taipei, Taiwan). The primers for the repeat copy number were $10 \mu \mathrm{M}$ telomere forward (5'-

140 CGGtTtGtTtGGGTTtGGGTtTGGGTTTG GGTTTGGGTT-3’) and $10 \mu \mathrm{M}$ telomere 
reverse (5'-GGCTTGCCTTACCCTTACCCTTACCCTTACCC TTACCCT-3') and for single-

143 3') and $10 \mu \mathrm{M}$ 36B4-reverse (5'-CCCATTCTATCATCAACGGGTACAA-3'). The profile of

144 the telomere and single copy gene amplification started with $95^{\circ} \mathrm{C}$ incubation for $10 \mathrm{~min}$

145 followed by 40 cycles of $15 \mathrm{sec}$ at $95^{\circ} \mathrm{C}$ and $1 \mathrm{~min}$ at $54^{\circ} \mathrm{C}$.

147 Biosystems, Foster City, CA, USA). Each PCR sample was run in duplicate using 3.1 ng DNA

148 per $10 \mu \mathrm{L}$ reaction. The targeted gene (Tel, ND1) PCR reaction was performed on spate runs

149 with the same samples, and melting curve result was expressed for every run to verify

150 specificity.

In vitro replicative lifespan and growth rate of $L F$ cells measured by cumulative population

doubling level (PDL) and population doubling rate (PDR)

At each subcultivation, confluent hypertrophic and non-hypertrophic LF cells were trysinized,

$156=\left[\log _{10}\left(\mathrm{~N}_{\mathrm{H}}\right)-\log _{10}\left(\mathrm{~N}_{\mathrm{I}}\right)\right] / \log _{10}(2)$, where $\mathrm{N}_{\mathrm{H}}=$ the number of harvested cells and $\mathrm{N}_{\mathrm{I}}=$ the number

157 of seeded cells (Cristofalo et al., 1998). The calculated population doubling increase was then

158 included in the previous population doubling level (PDL), to yield the cumulative population 
159 doubling level. Moreover, to determine growth rate, the PDRs of hypertrophic and non-

160 hypertrophic LF cells cultured in vitro were measured as follows: PDR $=$ the PDL/the number of

161 days in culture (Stenderup et al., 2003).

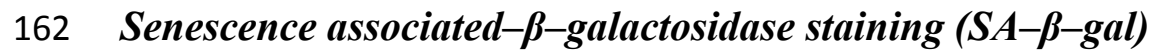

163 At each subcultivation, hypertrophic and non-hypertrophic LF cells were taken for SA- $\beta$-gal

164 staining. The percentage of SA- $\beta$-gal-positive LF cells was determined by the previous method

165 (Kim et al., 2009). Briefly, LF cells were seeded in 6-well plates at a cell density of $5.0 \times 10^{4}$

166 cells per well containing complete medium and incubated at $37^{\circ} \mathrm{C}$ for 24 hours. Cells were

167 washed in phosphate buffer saline (PBS) and fixed with $2 \%$ formaldehyde and $0.2 \%$

168 glutaraldehyde in 10X PBS for 15 minutes at room temperature. Then, cells were rinsed with

169 PBS and added SA- $\beta$-gal staining solution (Cell Signaling Technology, Danvers, MA, USA)

170 that contained $40 \mathrm{mM}$ citric acid/sodium phosphate ( $\mathrm{pH} 6.0$ ), $150 \mathrm{mM} \mathrm{NaCl}, 2 \mathrm{mM} \mathrm{mgCl}, 5 \mathrm{mM}$

171 potassium ferrocyanide, $5 \mathrm{mM}$ potassium ferricyanide, and $1 \mathrm{mg} / \mathrm{mL}$ of 5-bromo-4-chloro-3-

172 indoyl- $\beta-\mathrm{D}-$ galactopyranoside at $37^{\circ} \mathrm{C}$ for 16 hours. After incubation, $300 \mathrm{LF}$ cells in random

173 fields were counted and the percentage of SA- $\beta$-gal-positive LF cells was calculated by using

174 the following formula: the number of SA- $\beta-$ gal-positive LF cells/300 LF cells $) \times 100$. Cells

175 count was repeated twice and the mean was calculated. 
177 Total DNA was extracted directly from LF tissue. Firstly, LF tissues approximately $200 \mathrm{mg}$ was

178 homogenized with liquid nitrogen and added with $10 \mu 1$ proteinase $\mathrm{K}$ and $400 \mu 1$ lysis buffer

179 containing $50 \mathrm{mM}$ tris-hydrochloride ( $\mathrm{pH} 7.4), 1 \mathrm{mM}$ ethylenediaminetetraacetic ( $\mathrm{pH} 8.0), 0.5 \%$

$180 \mathrm{w} / \mathrm{v}$ sodium dodecylsulfate and incubated at $50^{\circ} \mathrm{C}$ for 2 hours. After incubation, homogenous LF

181 tissue was added with $250 \mu \mathrm{L}$ phenol and $250 \mu \mathrm{L}$ chloroform:indole-3-acetic acid $\left(\mathrm{CHCl}_{3}: \mathrm{IAA}_{\text {; }}\right.$

182 49:1). The lysate was centrifuged at $13,500 \mathrm{rpm}$ for 30 minutes at $4^{\circ} \mathrm{C}$. The supernatant was

183 carefully and transferred into a tube containing $4 \mu \mathrm{L}$ glycogen, $40 \mu \mathrm{L}$ sodium acetate, $800 \mu \mathrm{L}$

184 absolute ethanol and kept it overnight at $-20^{\circ} \mathrm{C}$. The lysate was centrifuged at $13,500 \mathrm{rpm}$ for 30

185 minutes at $4^{\circ} \mathrm{C}$. The supernatant was washed with $1 \mathrm{~mL} 70 \%$ ethanol and centrifuged at 13,500

$186 \mathrm{rpm}$ for 5 minutes at $4^{\circ} \mathrm{C}$. The supernatant was transferred into microcentrifuge tube and put into

187 vacuum machine for 15 minutes and dissolved with distilled water. Total DNA concentration

188 was determined using NanoDrop ${ }^{\circledR}$ ND-100 Spectrophotometer (Scientific, USA) and adjusted

189 DNA $200 \mu \mathrm{g} / \mathrm{mL}$ in each sample. The $8-\mathrm{OHdG}$ concentration of LF tissue was performed by

190 quantization of 8-OHdG in LF tissues DNA using HT 8-oxo-dG enzyme-linked immunosorbent

191 assay kit (Trivigen, Gaithersburg, MD, USA) according to the manufacturer's instructions.

\section{Statistical Analysis}

193 All statistical analyses were performed with the SPSS statistical package, version 20.0 (SPSS

194 Inc., Chicago, IL, USA). All values were reported as mean \pm standard error of the mean (SEM). 
195 Paired $t$-test was used to compare the means of two dependent groups. Comparison between the

196 two groups was employed by the Wilcoxon signed-rank test when the distributions were not

197 among the groups. A $P$-value less than 0.05 (based on a two-tailed test) was considered

198 statistically significant.

199

200 Results

201

RTL and 8-hydroxy-2'-deoxyguanosine levels of LF tissue samples in LSS patients

202 We investigated RTL in 48 non-hypertrophic and 48 hypertrophic LF tissues from LSS patients.

203 As shown in Figure 1, RTL in LF tissue samples were significantly lower in hypertrophic LF

204 tissue than in non-hypertrophic LF tissue $(1.01 \pm 0.07$ vs. $1.15 \pm 0.08 ; P=0.04)$. To investigate

205 status of oxidative damage in LSS patients, we determined 8-OHdG levels in 30 non-

206 hypertrophic and 30 hypertrophic LF tissue samples in LSS patients. The mean 8-OHdG levels

207 of LF tissue was significantly higher in hypertrophic LF tissue than non-hypertrophic LF tissue

$208(0.43 \pm 0.03$ vs. $0.64 \pm 0.09 ; P=0.02)$, as demonstrated in Figure 2 .

209

RTL in LF fibroblast cells

210 Real time-PCR analysis was used to determine the RTL of the non- hypertrophic and

211 hypertrophic LF fibroblast cells obtained from patients of 61, 66, and 77 years old. There was a 
212 significant decline in telomere length with advancing passages (Figure 3). RTL of the

213 hypertrophic LF fibroblast cells was significantly lower than that of the non-hypertrophic LF

214 fibroblast cells in the first passage ( $4.17 \pm 0.43$ vs. $5.85 \pm 0.19 ; P=0.04)$.

\section{Cumulative PDL and PDR}

216 The mean PDL of hypertrophic and non-hypertrophic LF cells obtained from patient aged 66

217 years were $4.84 \pm 0.40$ and $4.44 \pm 0.51$, respectively (Supplementary Fig. 1). The mean PDR of

218 hypertrophic and non-hypertrophic LF cells were $0.14 \pm 0.03$ and $0.12 \pm 0.02 \mathrm{PD} / \mathrm{day}$,

219 respectively (Supplementary Fig. 2).

\section{SA-B-gal staining}

221 The mean of percentages of non-hypertrophic and hypertrophic SA- $\beta$-gal-positive LF fibroblast

222 cells from patients aged 61 and 66 years at passage 1 were $0.30 \%, 0.00 \%, 0.30 \%$, and $0.30 \%$

223 respectively, which steadily increased with advancing passages (Figure 4). Meanwhile, The

224 mean of percentages of non-hypertrophic and hypertrophic SA- $\beta$-gal-positive LF fibroblast

225 cells from patients aged 77 years, even at passage 1 , were $2.70 \%$, and $6.30 \%$, which reached

226 more than $80 \%$ at passage 10 . The SA- $\beta$-gal staining study showed that SA- $\beta$-gal-positive LF

227 fibroblast cells was increased in hypertrophic LF compared to non-hypertrophic LF fibroblast

228 cells (Figure 5). 
231 Lumbar spinal stenosis is a common cause of low back pain caused by degenerative changes that

232 are common in older adults. The pathophysiology consists in compression within the central

233 canal and in changes in facet joints, disc bulging, and hypertrophic LF (Azimi et al., 2015).

234 Previous studies have shown that the thickness LF were associated with decrease of elastic and collagen fibers and increase of stiffness and fibrosis of LF (Park et al., 2005; Chen et al., 2014). miR-155 has been recently demonstrated to increase the expression of type I and type III collagen in fibroblasts of LF and correlate with the degree of fibrosis of LF in LSS patients

238 (Chen et al., 2014). non-hypertrophic and hypertrophic LF tissues and cell fibroblasts in LSS patients and evaluated senescent cells by SA- $\beta$-gal marker. To the best our knowledge, this is the first study to show

242 RTL in hypertrophic LF tissue in LSS patients. We showed that hypertrophic LF tissue from LSS patients presented shorter RTL than non-hypertrophic LF tissue, suggesting that the hypertrophic

244 LF cells may exhibit high turnover rate of cell division (Supplementary Fig. 1). This finding is accordant with the recent study, La Maitre and colleagues found that the mean telomere length was significantly decreased in subjects with degenerative disc disease when compared with non-

247 degenerative discs (Le Maitre et al., 2007). We hypothesized that the rate of telomere shortening 
248 in LF fibroblasts would reflect the rate of cell turnover in the human LF tissues (Supplementary

249 Fig. 2).

250

In the present study, LF fibroblast cells exhibited different RTL compared to the parental

251 LF tissues from which they have been isolated. The mechanism of shortened RTL in

252 hypertrophic LF tissues is not easily addressed. A number of possible mechanisms either

253 independently or in combination, may be postulated. In LSS, hypertrophic LF tissues contain

254 various kinds of cells including fibroblasts, inflammatory cells, and mesenchymal stem cells.

255 Recently, LF precursor cells were recently isolated from the hypertrophic LF tissues and these

256 cells displayed features of mesenchymal stem cells (Chen et al., 2011). All these cell types

257 in mixed populations have different telomere lengths. Our study relied upon average RTL in cell

258 populations or the entire tissue and the results would be masked when mixed populations in LF

259 tissues are measured. Moreover, chronic repetitive inflammation and oxidative stress

260 could contribute to telomere shortening in hypertrophic LF tissues. It should be noted that a

261 variety of these factors may act in concert to generate the phenomenon. The mechanism behind

262 the connection of different RTL in hypertrophic LF tissue and in hypertrophic LF fibroblast cells

263 remains a mystery and requires further study.

264 Telomere erosion and DNA damage are the most important of aging-related factors.

265 Environmental stimuli in degenerative diseases may also be causing cell senescence. In our 
266 study, senescence marker analysis revealed greater numbers of SA- $\beta$-gal positive cells in

267 hypertrophic LF fibroblastic cells with advancing culture passages of elderly patients with LSS.

268 These findings are also in line with a previous study by Jeong and coworkers (Jeong et al.,

269 2014), where disc cells from older patients had a lower telomere length and reached to

270 senescence in vitro earlier than those from younger patients with intervertebral disc disease. The

271 results indicate that patient age is major factor. Cells from elderly patients might have lower

272 levels of telomerase activity and senescent cells accumulate with age.

An increased generation of ROS and radicals in cellular redox level has been associated

274 with degenerative diseases and aging (Venkatesh et al., 2012). The 8-OHdG marker is measured

275 as an indicator of oxidative DNA damage. The current study showed that hypertrophic LF 8-

276 OHdG values were significantly higher in hypertrophic LF than in non-pathologic LF. This

277 finding is consistent with a previous study (Tsai et al., 2010) showing that cultured orbital

278 fibroblasts from patients with Graves' ophthalmopathy had higher levels of 8-OHdG compared

279 with normal controls. In our recent study (Udomsinprasert et al., 2016), we revealed that 8-

280 OHdG levels were significantly increased in the serum and liver specimens of biliary atresia

281 patients compared with controls. The increasing of oxidative stress condition may be causing

282 DNA damage within cells that involved in the pathogenesis of hypertrophic LF in LSS patients. 
284 length and oxidative DNA damage in LSS patients, but did not measure the activity of

285 telomerase enzymes. The mechanisms underlying altered telomerase expression in the

286 hypertrophic LF remain unclear. Additional studies are warranted to further investigate the

287 telomerase activity and SA- $\beta$-gal staining in LF fibroblast cells from the younger and older

288 patients with LSS. Secondly, this study was cross-sectional in its design. Therefore, prospective

289 longitudinal researches are necessary to demonstrate the association between RTL, 8-OHdG,

290 and LF thickness. Moreover, we could not access normal LF tissues or LF fibroblasts from

291 normal age-matched subjects without LSS, or any other normal comparable tissues, that would

292 serve as a good control to determine RTL and 8-OHdG levels. Lastly, the sample size was

293 relatively small, which made it challenging to analyze data with different age decades of patients.

294 Our power of statistical difference was relatively low. Thus, further studies with large numbers

295 of patients are warranted.

\section{Conclusions}

297 The present study demonstrated that patients with LSS had a shorter RTL and higher oxidative

298 DNA damage in hypertrophic LF than in non-pathologic LF. The in vitro experiments showed

299 that the number of senescence cells was increased in the hypertrophic LF fibroblasts. Although 
300 underlying mechanisms and pathways remain unclear and require additional studies. This study

301 may shed some light on the pathogenesis of hypertrophic LF in lumbar spinal stenosis.

302

303 Abbreviation

304 LSS Lumbar spinal stenosis

305 LF Ligamentum flavum

306 8-OHdG 8-hydroxy-2'-deoxyguanosine

307 SA- $\beta$-gal senescence-associated $\beta$-galactosidase

\section{Acknowledgements}

309 The author would also like to thank the Research Core Facility of the Department of

310 Biochemistry and Chulalongkorn Medical Research Center (ChulaMRC) for kindly providing

311 facilities. The authors are gratefully thankful to Napaphat Jirathanathornnukul for technical

312 assistance. We thank Professor Henry Wilde for reviewing and proof-reading the manuscript. 


\section{References}

317

Azimi P, Mohammadi HR, Benzel EC, Shahzadi S, Azhari S. 2015. Lumbar Spinal Stenosis Classification Criteria: A New Tool. Asian Spine Journal. 9:399-406. DOI 10.4184/asj.2015.9.3.399

Cawthon RM. 2002. Telomere measurement by quantitative PCR. Nucleic Acids Res 30:e47.

Chen J, Liu Z, Zhong G, Qian L, Li Z, Qiao Z, Chen B, Wang H. 2014. Hypertrophy of ligamentum flavum in lumbar spine stenosis is associated with increased miR155 level. Dis Markers. 2014:786543. DOI 10.1155/2014/786543.

Chen MH, Hu CK, Chen PR, Chen YS, Sun JS, Chen MH. 2014. Dose-dependent regulation of cell proliferation and collagen degradation by estradiol on ligamentum flavum. $B M C$ Musculoskeletal Disorders. 15:238. DOI 10.1186/1471-2474-15-238

Chen YT, Wei JD, Wang JP, Lee HH, Chiang ER, Lai HC, Chen LL, Lee YT, Tsai CC, Liu CL, Hung SC. 2011. Isolation of mesenchymal stem cells from human ligamentum flavum: implicating etiology of ligamentum flavum hypertrophy. Spine (Phila Pa 1976). 36:E1193-200. DOI 10.1097/BRS.0b013e3182053f58.

Cristofalo VJ, Allen RG, Pignolo RJ, Martin BG, Beck JC. 1998. Relationship between donor age and the replicative lifespan of human cells in culture: a reevaluation. Proc Natl Acad Sci U S A 95:10614-10619. 
334 De Felice B, Wilson RR, Nacca M. 2009. Telomere shortening may be associated with human keloids. BMC Medical Genetics 10:110. DOI 10.1186/1471-2350-10-110.

336

337

Diotti R, Loayza D. 2011. Shelterin complex and associated factors at human telomeres. Nucleus 2:119-35. DOI 10.4161/nucl.2.2.15135

Dröge W. 2002. Free radicals in the physiological control of cell function. Physiological Reviews 82:47-95. DOI: 10.1152/physrev.00018.2001

Honsawek S, Poonpukdee J, Chalermpanpipat C, Payungporn S, Limthongkul W, Yingsakmongkol W, Thanakit V, Parkpian V. 2013. Hypertrophy of the ligamentum flavum in lumbar spinal canal stenosis is associated with increased bFGF expression. Int Orthop 37:1387-92. DOI: 10.1007/s00264-013-1864-y.

Houben JM, Moonen HJ, van Schooten FJ, Hageman GJ. 2008. Telomere length assessment: biomarker of chronic oxidative stress? Free Radical Biology and Medicine 44:235-46. DOI 10.1016/j.freeradbiomed.2007.10.001

Jeong SW, Lee JS, Kim KW. 2014. In vitro lifespan and senescence mechanisms of human nucleus pulposus chondrocytes. Spine Journal 14:499-504. DOI 10.1016/j.spinee.2013.06.099 
350 Katz JN, Harris MB. 2008. Clinical practice. Lumbar spinal stenosis. The New England Journal

351 of Medicine 358:818-25. DOI 10.1056/NEJMcp0708097

352 Kim KW, Ha KY, Lee JS, Na KH, Kim YY, Woo YK. 2008. Senescence of nucleus pulposus

353

354

355

356

357

358

359

360

361

362

363

364

365

366

chondrocytes in human intervertebral discs. Asian Spine Journal 2:1-8. DOI

10.4184/asj.2008.2.1.1

Kim KW, Chung HN, Ha KY, Lee JS, Kim YY. 2009. Senescence mechanisms of nucleus pulposus chondrocytes in human intervertebral discs. Spine Journal 9:658-66. DOI 10.1016/j.spinee.2009.04.018

Kroese LJ, Scheffer PG. 2014. 8-hydroxy-2'-deoxyguanosine and cardiovascular disease: a systematic review. Current Atherosclerosis Reports 16:452. DOI 10.1007/s11883-0140452-y.

Le Maitre CL, Freemont AJ, Hoyland JA. 2007. Accelerated cellular senescence in degenerate intervertebral discs: a possible role in the pathogenesis of intervertebral disc degeneration. Arthritis Research and Therapy 9:R45. DOI 10.1186/ar2198

Li S, Xia H, Han C. 2015. Retrospective analysis on correlation factors of preserving the ligamentum flavum in microendoscopic discectomy. Clinical Neurology and Neurosurgery 139:46-50. DOI 10.1016/j.clineuro.2015.08.018 
367 Lingner J, Cech TR. 1998. Telomerase and chromosome end maintenance. Curr Opin Genet Dev

368

369

370

371

372

373

374

375

376

377

378

379

380

381

382

383
Losiniecki AJ, Serrone JC, Keller JT, Bohinski RJ. 2013. Lumbar ligamentum flavum: spatial relationships to surrounding anatomical structures and technical description of en bloc resection. Journal of Neurological Surgery. Part A, Central European Neurosurgery 74:388-92. DOI 10.1055/s-0033-1348350

Lu, F. 2007. Reactive oxygen species in cancer, too much or too little? Medical Hypotheses 69:1293-8. DOI 10.1016/j.mehy.2007.03.017

McEachern MJ, Krauskopf A, Blackburn EH. 2000. Telomeres and their control. Annu Rev Genet 34:331-358.

Mobbs RJ, Dvorak M. 2007. Ossification of the ligamentum flavum: diet and genetics. Journal of Clinical Neuroscience 14:703-5. DOI 10.1016/j.jocn.2006.01.010

O'Sullivan RJ, Karlseder J. 2010. Telomeres: protecting chromosomes against genome instability. Nat Rev Mol Cell Biol 11:171-81. DOI: 10.1038/nrm2848.

Park JB, Lee JK, Park SJ, Riew KD. 2005. Hypertrophy of ligamentum flavum in lumbar spinal stenosis associated with increased proteinase inhibitor concentration. The Journal of Bone and Joint Surgery. American Volume 87:2750-7. DOI 10.2106/JBJS.E.00251 
384 Park JB, Kong CG, Suhl KH, Chang ED, Riew KD. 2009. The increased expression of matrix

385

386

387

388

389

390

391

392

393

394

395

396

397

398

399 metalloproteinases associated with elastin degradation and fibrosis of the ligamentum flavum in patients with lumbar spinal stenosis. Clin Orthop Surg 1:81-9. DOI:

10.4055/cios.2009.1.2.81.

Park JO, Lee BH, Kang YM, Kim TH, Yoon JY, Kim H, Kwon UH, Lee KI, Lee HM, Moon SH. 2013. Inflammatory cytokines induce fibrosis and ossification of human ligamentum flavum cells. J Spinal Disord Tech 26:E6-12. DOI: 10.1097/BSD.0b013e3182698501.

Sajous L, Botta A, Sari-Minodier I. 2008. [Urinary 8-hydroxy-2'-deoxyguanosine: a biomarker of environmental oxidative stress?]. Annales De Biologie Clinique 66:19-29. DOI 10.1684/abc. 2008.0188

Stenderup K, Justesen J, Clausen C, Kassem M. 2003. Aging is associated with decreased maximal life span and accelerated senescence of bone marrow stromal cells. Bone 33:919-926. DOI 10.1016/j.bone.2003.07.005

Takahashi K, Nishida H, Takeda H, Shin K. 2004. Telomere length in leukocytes and cultured gingival fibroblasts from patients with aggressive periodontitis. Journal of Periodontology 75:84-90. DOI 10.1902/jop.2004.75.1.84 
400 Tsai CC, Wu SB, Cheng CY, Kao SC, Kau HC, Chiou SH, Hsu WM, Wei YH. 2010. Increased

401

402

403

404

405

406

407

408

409

410

411

412

413

414

oxidative DNA damage, lipid peroxidation, and reactive oxygen species in cultured orbital fibroblasts from patients with Graves' ophthalmopathy: evidence that oxidative stress has a role in this disorder. Eye (London, England) 24:1520-5. DOI 10.1038/eye.2010.31

Udomsinprasert W, Poovorawan Y, Chongsrisawat V, Vejchapipat P, Zhan D, Honsawek S. 2015. Telomere Length in Peripheral Blood Leukocytes Is Associated with Severity of Biliary Atresia. PLoS One 10:e0134689. DOI10.1371/journal.pone.0134689

Udomsinprasert W, Kitkumthorn N, Mutirangura A, Chongsrisawat V, Poovorawan Y, Honsawek S. 2016. Global methylation, oxidative stress, and relative telomere length in biliary atresia patients. Scientific Reports 6:26969. DOI 10.1038/srep26969

Valavanidis A, Vlachogianni T, Fiotakis C. 2009. 8-hydroxy-2'-deoxyguanosine (8-OHdG): A critical biomarker of oxidative stress and carcinogenesis. Journal of environmental science and health. Part C, Environmental carcinogenesis \& ecotoxicology reviews 27:120-39. DOI10.1080/10590500902885684 
415 Valko M, Izakovic M, Mazur M, Rhodes CJ, Telser J. 2004. Role of oxygen radicals in DNA

416

417

418

419

420

421

422

423

424

425

426

427

428

429

430

Clinical Science (London) 120:427-40. DOI 10.1042/CS20100385

10.1016/j.bbamcr.2011.11.003

10.1002/jor.21431.
ATP-dependent Lon protease. Biochimica et Biophysica Acta 1823:56-66. DOI

Yabe Y, Hagiwara Y, Ando A, Tsuchiya M, Minowa T, Takemura T, Honda M, Hatori K,

Sonofuchi K, Kanazawa K, Koide M, Sekiguchi T, Itoi E. 2015. Chondrogenic and

fibrotic process in the ligamentum flavum of patients with lumbar spinal canal stenosis.

Spine (Phila Pa 1976) 40:429-35. DOI10.1097/BRS.0000000000000795

Zhong ZM, Zha DS, Xiao WD, Wu SH, Wu Q, Zhang Y, Liu FQ, Chen JT. 2011.

Hypertrophy of ligamentum flavum in lumbar spine stenosis associated with the

increased expression of connective tissue growth factor. J Orthop Res 29:1592-7. DOI:

Zhu H, Belcher M, van der Harst P. 2011. Healthy aging and disease: role for telomere biology?

431 
Figure 1

Comparison of relative telomere length of non-pathologic and pathologic ligamentum flavum tissues from LSS patients.

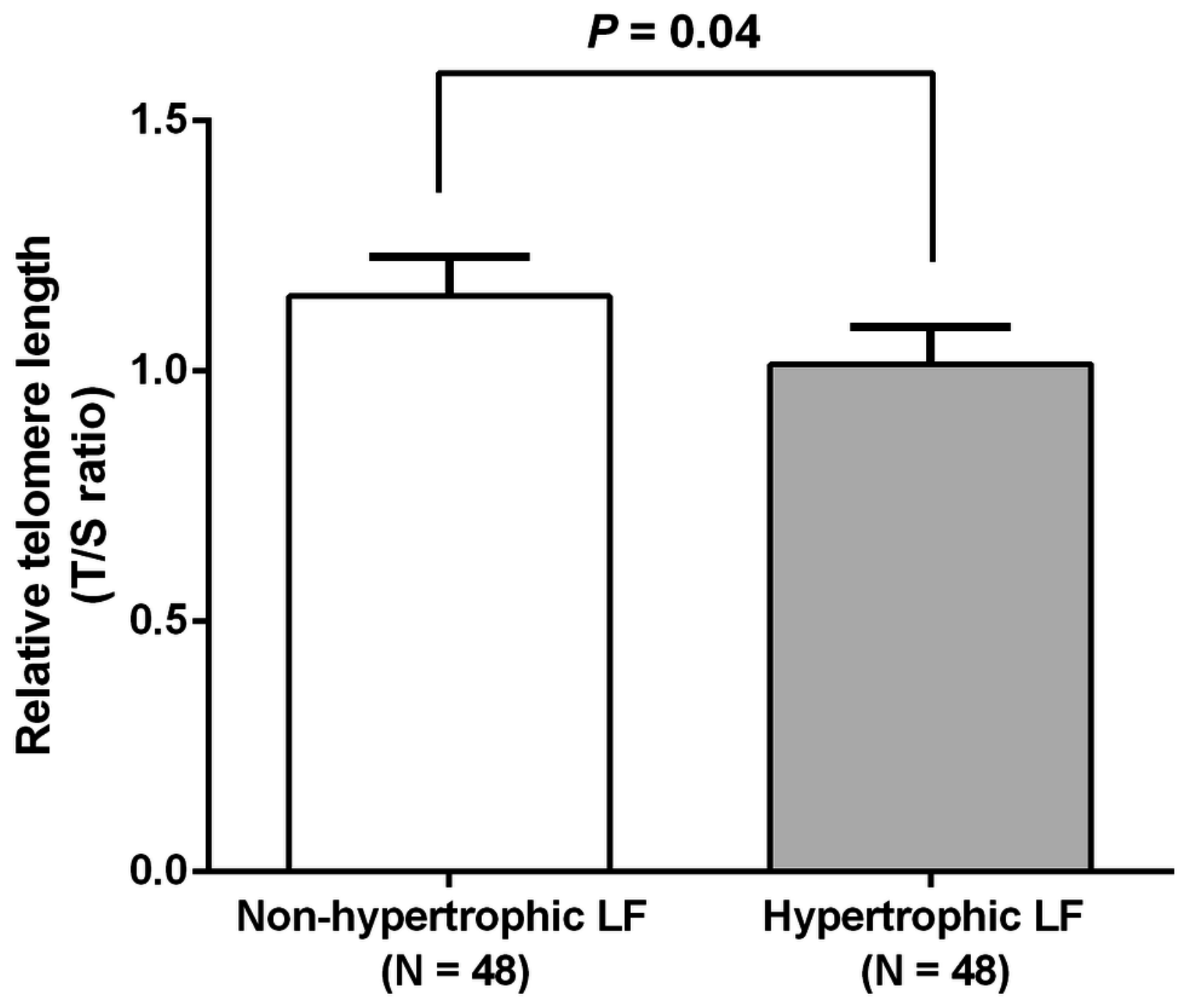


Figure 2

Ligamentum flavum tissue 8-hydroxy-2'-deoxyguanosine concentration of nonpathologic and pathologic ligamentum flavum tissues from LSS patients.

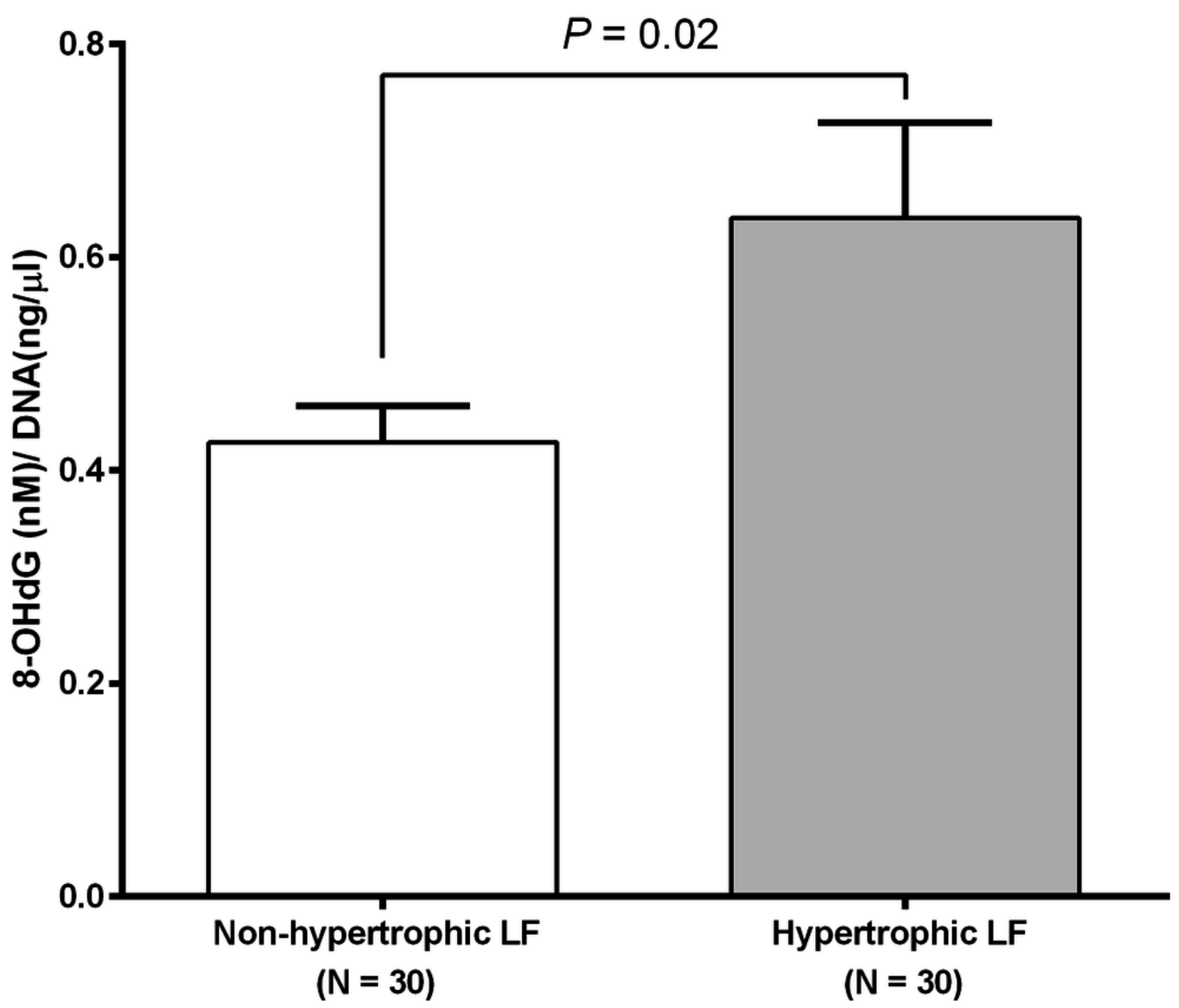


Figure 3

Relative telomere length of non-pathologic and pathologic LF fibroblasts cells from LSS patients aged 61,66 , and 77 years.

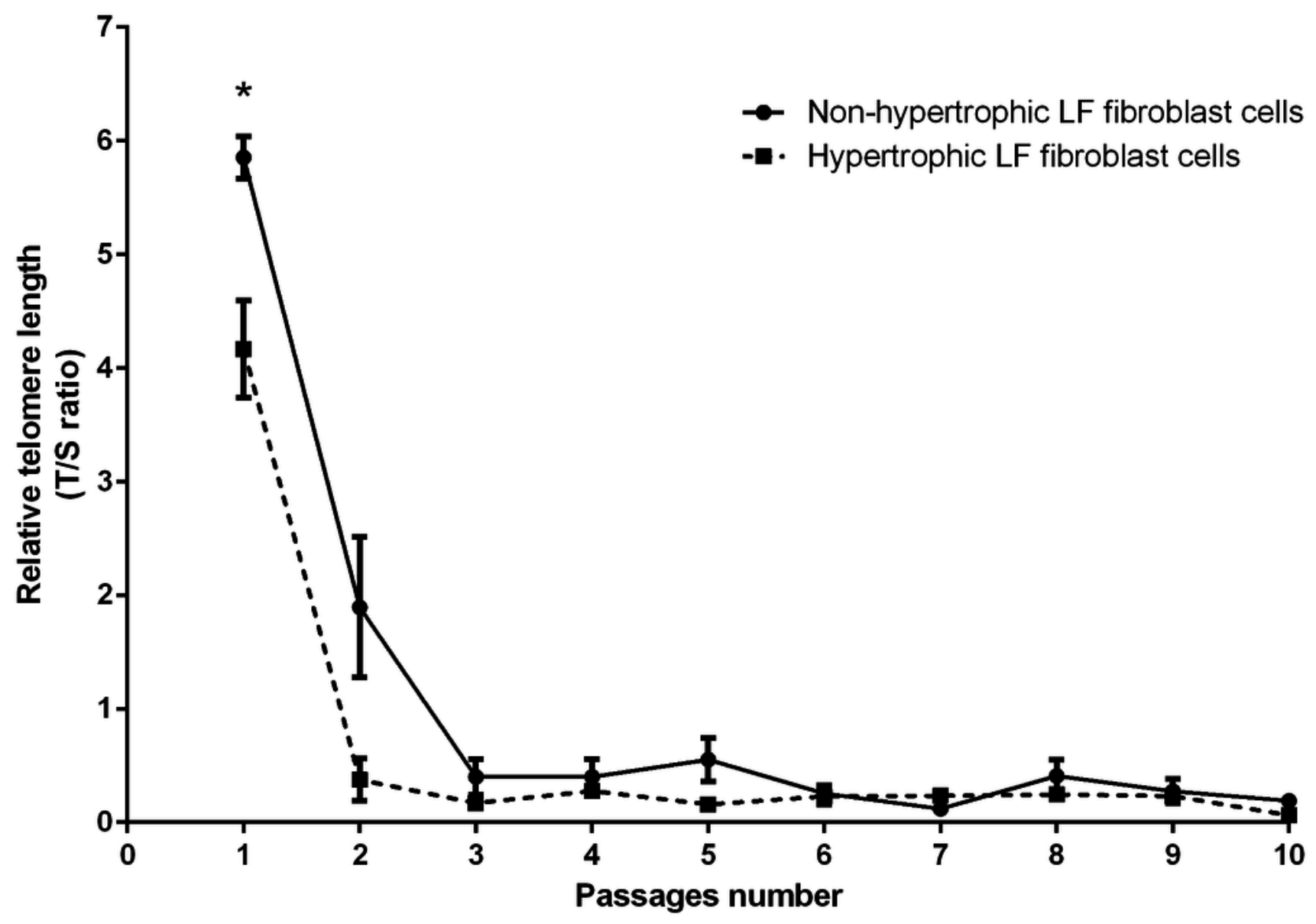


Figure 4

The percentage of the senescence-associated- $\beta$-galactosidase (SA- $\beta$-gal) positive of non-pathologic and pathologic LF fibroblasts cells from LSS patients aged 61, 66, and 77 years.

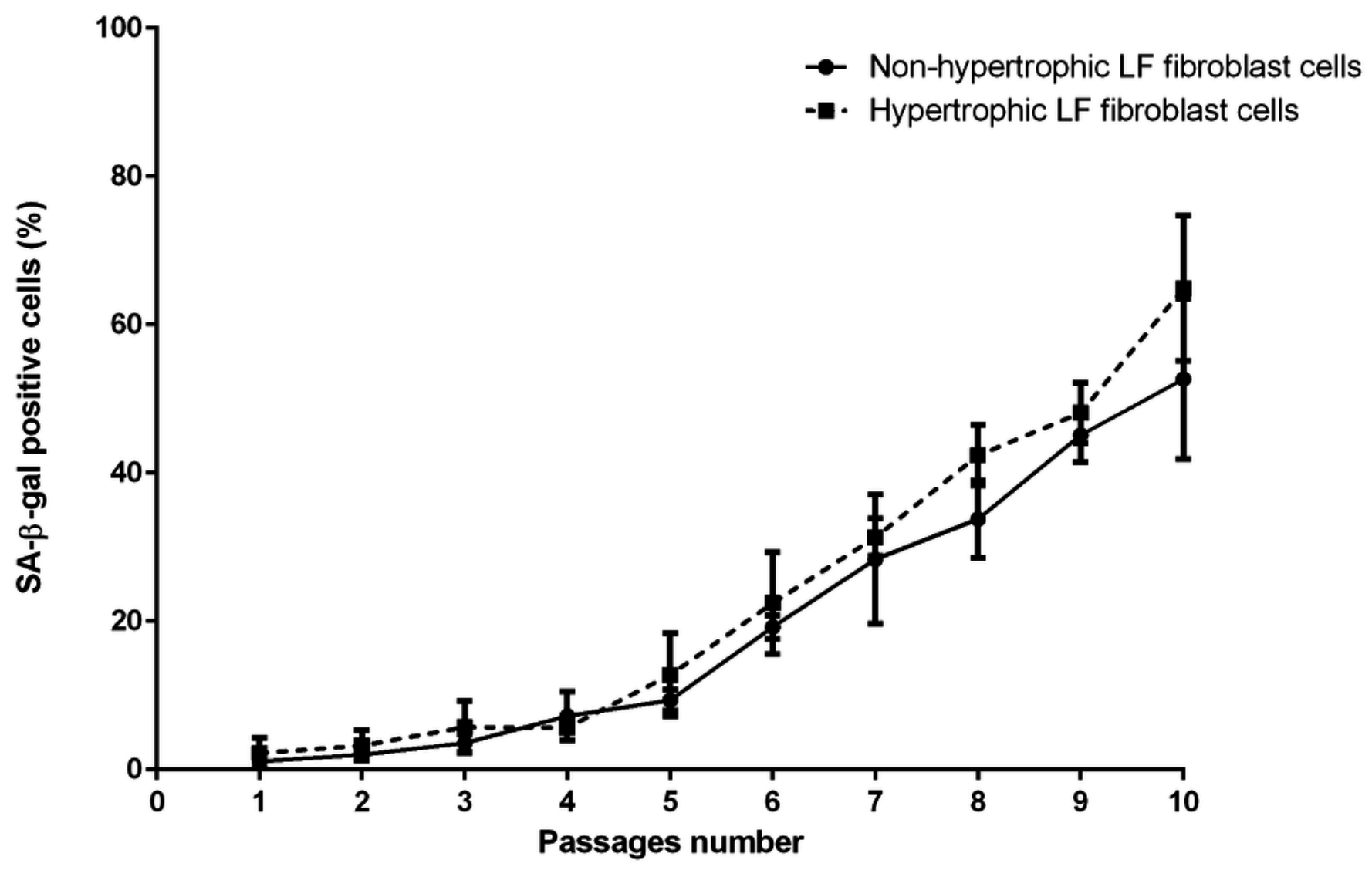




\section{Figure 5}

The senescence senescence-associated- $\beta$-galactosidase (SA- $\beta$-gal) positive pathologic LF fibroblasts cells as shown in arrows.

Representative staining from patients aged 61 years (A) passage 1; (B) passage 10 and patients aged 77 years (C) passage 1; (D) passage 10.
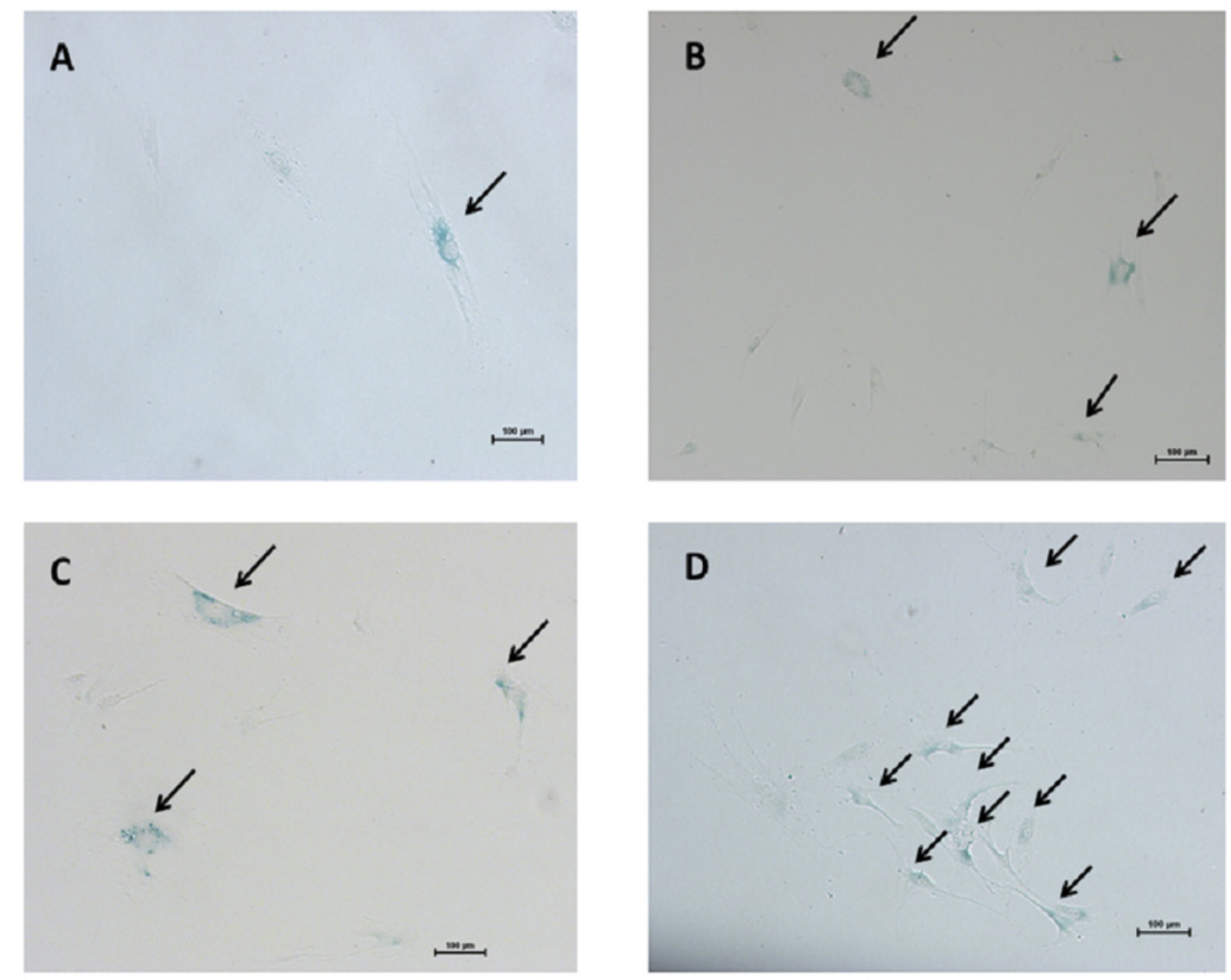